\title{
An Example of a New Type of Cosmological Solutions of Einstein's Field Equations of Gravitation
}

\author{
KURT GÖDEL \\ Institute for Advanced Study, Princeton, New Jersey
}

\section{THE MAIN PROPERTIES OF THE NEW SOLUTION}

A LL cosmological solutions with non-vanishing denA sity of matter known at present ${ }^{1}$ have the common property that, in a certain sense, they contain an "absolute" time coordinate, ${ }^{2}$ owing to the fact that there exists a one-parametric system of three-spaces everywhere orthogonal on the world lines of matter. It is easily seen that the non-existence of such a system of three-spaces is equivalent with a rotation of matter relative to the compass of inertia. In this paper I am proposing a solution (with a cosmological term $\neq 0$ ) which exhibits such a rotation. This solution, or rather the four-dimensional space $S$ which it defines, has the further properties:

(1) $S$ is homogeneous, i.e., for any two points $P, Q$ of $S$ there exists a transformation of $S$ into itself which carries $P$ into $Q$. In terms of physics this means that the solution is stationary and spatially homogeneous.

(2) There exists a one-parametric group of transformations of $S$ into itself which carries each world line of matter into itself, so that any two world lines of matter are equidistant.

(3) $S$ has rotational symmetry, i.e., for each point $P$ of $S$ there exists a one parametric group of transformations of $S$ into itself which carries $P$ into itself.

(4) The totality of time-like and null vectors can be divided into + - and --vectors in such a way that: (a) If $\xi$ is a +-vector, $-\xi$ is a - -vector, (b) a limit of +- (or - -) vectors, if $\neq 0$, is again $a+-$ (or -- ) vector. That is, a positive direction of time can consistently be introduced in the whole solution.

After a direction of time has been introduced in this way, a temporal orientation is defined for the world line of every (real or possible) particle of matter or light, i.e., it is determined for any two neighboring points on it which one is earlier. On the other hand, however, no uniform temporal ordering of all point events, agreeing in direction with all these individual orderings, exists. This is expressed in the next property:

(5) It is not possible to assign a time coordinate $t$ to each space-time point in such a way that $t$ always increases, if one moves in a positive time-like direction;

\footnotetext{
${ }^{1}$ See, for example, H. P. Robertson, Rev. Mod. Phys. 5, 62 (1933).

${ }^{2}$ As to the philosophical consequences which have been drawn from this circumstance see J. Jeans, "Man and the Universe," Halley Stewart Lecture (1935), and my article forthcoming in the Einstein volume of the Library of Living Philosophers.
}

and this holds both for an open and a closed time coordinate.

(6) Every world line of matter occurring in the solution is an open line of infinite length, which never approaches any of it's preceding points again; but there also exist closed time-like lines. ${ }^{3}$ In particular, if $P, Q$ are any two points on a world line of matter, ${ }^{4}$ and $P$ precedes $Q$ on this line, there exists a time-like line connecting $P$ and $Q$ on which $Q$ precedes $P$; i.e., it is theoretically possible in these worlds to travel into the past, or otherwise influence the past.

(7) There exist no three-spaces which are everywhere space-like and intersect each world line of matter in one point.

(8) If $\sum$ is any system of mutually exclusive threespaces, each of which intersects every world line of matter in one point, ${ }^{5}$ then there exists a transformation which carries $S$ and the positive direction of time into itself, but does not carry $\sum$ into itself; i.e., an absolute time does not exist, even if it is not required to agree in direction with the times of all possible observers (where "absolute" means: definable without reference to individual objects, such as, e.g., a particular galactic system).

(9) Matter everywhere rotates relative to the compass of inertia with the angular velocity: $2(\pi \kappa \rho)^{\frac{1}{2}}$, where $\rho$ is the mean density of matter and $\kappa$ Newton's gravitational constant.

\section{DEFINITION OF THE LINEAR ELEMENT AND PROOF THAT IT SATISFIES THE FIELD EQUATIONS}

The linear element of $S$ is defined by the following expression : ${ }^{6}$

$$
a^{2}\left(d x_{0}^{2}-d x_{1}^{2}+\left(e^{2 x_{1}} / 2\right) d x_{2}{ }^{2}-d x_{3}^{2}+2 e^{x_{1}} d x_{0} d x_{2}\right),
$$

${ }^{3}$ If the tangent of a line is discontinuous, the line is to be considered as time-like only if the corners can be so rounded off, that the resulting line is everywhere time-like.

4 "World line of matter" without further specification always refers to the world lines of matter occurring as such in the solution under consideration.

${ }^{5}$ Another hypothesis about $\Sigma$ under which the conclusion holds is that $\Sigma$ is one-parametric and oriented (where the orientation refers to the space whose points are the elements of $\Sigma$ ).

${ }^{6}$ This quadratic form can also be written thus

$$
a^{2}\left[\left(d x_{0}+e^{x_{1}} d x_{2}\right)^{2}-d x_{1}^{2}-\frac{e^{2 x_{1}}}{2} d x_{2}{ }^{2}-d x_{3}^{2}\right],
$$

which makes it evident that, as required, its signature is everywhere -2 . The three-space obtained by leaving out the term $-d x_{3}{ }^{2}$ has a simple geometric meaning (see below). Essentially the 
where $a$ is a positive number. The matrices of the $g_{i k}$ and $g^{i k}$, therefore, are the two:

$a^{2} \cdot\left\|\begin{array}{cccr}1 & 0 & \mathrm{e}^{x_{1}} & 0 \\ 0 & -1 & 0 & 0 \\ e^{x_{1}} & 0 & e^{2 x_{1} / 2} & 0 \\ 0 & 0 & 0 & -1\end{array}\right\|, \quad \frac{1}{a^{2}} \cdot\left\|\begin{array}{rccr}-1 & 0 & 2 e^{-x_{1}} & 0 \\ 0 & -1 & 0 & 0 \\ 2 e^{-x_{1}} & 0 & -2 e^{-2 x_{1}} & 0 \\ 0 & 0 & 0 & -1\end{array}\right\|$.

Owing to the fact that only two of the forty $\partial g_{i k} / \partial x_{l}$ are $\neq 0$, namely $\partial g_{22} / \partial x_{1}$ and $\partial g_{02} / \partial x_{1}$, the $\Gamma_{i, k l}$ and $\Gamma_{k l}^{i}$ can very easily be computed. One obtains the values:

$$
\begin{aligned}
\Gamma_{0,12} & =-\Gamma_{1,02}=\Gamma_{2,01}=\left(a^{2} / 2\right) e^{x_{1}}, \\
\Gamma_{1,22} & =-\Gamma_{2,12}=-\left(a^{2} / 2\right) e^{2 x_{1}}, \\
\Gamma_{01} 0 & =1, \quad \Gamma_{12}=\Gamma_{02}=e^{x_{1}} / 2, \\
\Gamma_{22}{ }^{1} & =e^{2 x_{1}} / 2, \quad \Gamma_{01}{ }^{2}=-e^{-x_{1}} .
\end{aligned}
$$

These $\Gamma_{i, k l}$ and $\Gamma_{k l}$, and those obtained from them by interchanging the last two (or the lower two) indices are the only ones that do not vanish.

Using for $R_{i k}$ the formula ${ }^{7}$

$$
R_{i k}=\frac{\partial}{\partial x_{\sigma}} \Gamma_{i k}{ }^{\sigma}-\frac{1}{2} \frac{\partial^{2} \log g}{\partial x_{i} \partial x_{k}}+\frac{1}{2} \Gamma_{i k}{ }^{\sigma} \frac{\partial \log g}{\partial x_{\sigma}}-\Gamma_{\sigma i}{ }^{\rho} \Gamma_{\rho k}{ }^{\sigma},
$$

and taking account of the fact that $\partial / \partial x_{i}$, except for $i=1$, vanishes for every magnitude of the solution, and that $g=\left(a^{8} / 2\right) e^{2 x_{1}}$, we obtain

$$
R_{i k}=\frac{\partial}{\partial x_{1}} \Gamma_{i k^{1}}+\Gamma_{i k^{1}}-\Gamma_{\rho i}{ }^{\sigma} \Gamma_{\sigma k^{\rho}}
$$

This yields the values for the $R_{i k}$

$$
R_{00}=1, \quad R_{22}=e^{2 x_{1}}, \quad R_{02}=R_{20}=e^{x_{1}} ;
$$

all other $R_{i k}$ vanish. Hence,

$$
R=1 / a^{2} .
$$

The unit vector $u$ in the direction of the $x_{0}$-lines has the contravariant components $1 / a, 0,0,0$ and, therefore, the covariant components $a, 0, a e^{x_{1}}, 0$.

Hence, we obtain:

$$
R_{i k}=1 / a^{2} \cdot u_{i} u_{k} .
$$

Since, furthermore, $R$ is a constant, the relativistic field equations (with the $x_{0}$-lines as world lines of matter), i.e., the equations ${ }^{8}$

$$
R_{i k}-\frac{1}{2} g_{i k} R=8 \pi \kappa \rho u_{i} u_{k}+\lambda g_{i k}
$$

same three-space, but with the signature +3 and with more general values of the constants, has been investigated in connection with the theory of continuous groups, without any reference to relativity theory. See, for example, L. Bianchi, Lezioni sulla teoria dei gruppi continui finiti di transformazioni (Pisa, 1918), p. 565.

7 Note that physicists frequently denote with $-R_{i k}$ what is here denoted with $R_{i k}$, with a corresponding change of sign in the field equations.

${ }^{8}$ The linear element is supposed to give time-like distances in seconds and space-like distances in light seconds. Therefore, the coefficient of $u_{i} u_{k}$ differs from the usual one by a factor $c^{2}$.

are satisfied (for a given value of $\rho$ ), if we put

$$
1 / a^{2}=8 \pi \kappa \rho, \quad \lambda=-R / 2=-1 / 2 a^{2}=-4 \pi \kappa \rho .
$$

The sign of the cosmological constant here is the opposite of that occurring in Einstein's static solution. It corresponds to a positive pressure.

\section{PROOFS FOR THE PROPERTIES ENUMERATED}

That there exists no-one parametric system of threespaces orthogonal on the $x_{0}$-lines follows immediately from the necessary and sufficient condition which a vector field $v$ in a four-space must satisfy, if there is to exist a system of three-spaces everywhere orthogonal on the vectors of the field. This condition requires that the skew symmetric tensor

$$
a_{i k l}=v_{i}\left(\frac{\partial v_{k}}{\partial x_{l}}-\frac{\partial v_{l}}{\partial x_{k}}\right)+v_{k}\left(\frac{\partial v_{l}}{\partial x_{i}}-\frac{\partial v_{i}}{\partial x_{l}}\right)+v_{l}\left(\frac{\partial v_{\imath}}{\partial x_{k}}-\frac{\partial v_{k}}{\partial x_{i}}\right)
$$

should vanish identically. The components of the corresponding vector

$$
w^{j}=\frac{\epsilon^{j i k l}}{6 \cdot \sqrt{g}} a_{i k l}
$$

however, in our case (i.e., for $v_{i}=u_{i}$ ) have the values $0,0,0, \sqrt{2} / a^{2}$. The non-vanishing of $w^{3}$ shows, moreover, that there exist no surfaces orthogonal on the $x_{0}$-lines in the subspaces $x_{3}=$ const.

If $v$ is the unit vector representing the velocity of matter, the vector $w$ (which evidently is always orthogonal to $v$ ) is twice the angular velocity of matter in a local inertial system in whose origin matter is at rest at the moment considered. ${ }^{9}$ Hence, property (9) follows at once.

The properties (1) and (2) follow from the directly verifiable fact that the space $S$ admits the following four systems of transformations into itself,

(I)

$$
x_{0}=x_{0}{ }^{\prime}+b
$$$$
x_{i}=x_{i}{ }^{\prime} \text { for } i \neq 0
$$$$
\text { (III) } x_{3}=x_{3}{ }^{\prime}+b
$$$$
\text { (II) } x_{2}=x_{2}^{\prime}+b
$$$$
x_{i}=x_{i}^{\prime} \text { for } i \neq 2
$$$$
x_{i}=x_{i}^{\prime} \text { for } i \neq 3
$$

(IV) $x_{1}=x_{1}{ }^{\prime}+b$

$x_{2}=x_{2}^{\prime} e^{-b}$

$x_{0}=x_{0}{ }^{\prime}$

$x_{3}=x_{3}{ }^{\prime}$

where $b$ is an arbitrary real number.

A division of the time-like and null vectors into +and - - vectors as required by (4) can be effected by defining $\xi$ to be a + - or a --vector according as to whether the inner product $(\xi u)=g_{i k} \xi^{i} u^{k}$ is $>$ or $<0$.

In order to prove (3) we introduce new coordinates $r, \varphi, t, y$ (where $r, \varphi, t$ are cylindrical coordinates in the subspaces $x_{3}=$ const., and $y$, up to a constant factor, is $=x_{3}$ ) by the following formulas of transformation,

${ }^{9}$ This is an immediate consequence of the definition of a local inertial system, which requires that $g_{i k}= \pm \delta_{k}^{i}$ and $\partial g_{i k} / \partial x_{l}=0$ for every $i, k, l$, 
which are easily solvable with respect to the $x_{i}$,

$$
\begin{aligned}
e^{x_{1}} & =\operatorname{ch} 2 r+\cos \varphi \operatorname{sh} 2 r \\
x_{2} e^{x_{1}} & =\sqrt{2} \sin \varphi \operatorname{sh} 2 r \\
\operatorname{tg}\left(\frac{\varphi}{2}+\frac{x-2 t}{2 \sqrt{2}}\right) & =e^{-2 r t g} \frac{\varphi}{2}, \quad \text { where } \quad\left|\frac{x_{0}-2 t}{2 \sqrt{2}}\right|<\frac{\pi}{2} \\
x_{3} & =2 y .
\end{aligned}
$$

This leads ${ }^{10}$ to the expression for the linear element,

$$
4 a^{2}\left(d t^{2}-d r^{2}-d y^{2}+\left(s h^{4} r-s h^{2} r\right) d \varphi^{2}+2 \sqrt{2} s h^{2} r d \varphi d t\right),
$$

which directly exhibits the rotational symmetry, since the $g_{i k}$ do not depend on $\varphi$.

Property (6) now follows easily: If $c$ is defined by $s h c=1$ (i.e., $c=\log (1+\sqrt{2})$ ), then for any $R>c$ we have $s h^{4} R-s h^{2} R>0$; hence, the circle defined by $r=R$, $t=y=0$ is everywhere time-like (the positive direction of time, by the above definition, being that of increasing $\varphi)$. Hence, the line defined by

$$
r=R, \quad y=0, \quad t=-\alpha \varphi \quad(0 \leq \varphi \leq 2 \pi)
$$

for sufficiently small $\alpha$ also will be everywhere time-like. However, the initial point $Q$ of this line (i.e., the point corresponding to $\varphi=0$ ) and the end point $P$ (i.e., that corresponding to $\varphi=2 \pi)$ are situated on the $t$-line: $r=R, y=\varphi=0$, and $P$ precedes $Q$ on this line if $\alpha>0$. Repeating this procedure any point preceding $Q$ on its $t$-line can be reached, and because of the homogeneity of the solution the same can be done for every point.

Property (7), in view of (2) and (4), is an immediate consequence of (6). For, a three-space satisfying the two conditions stated in (7) in conjunction with time measured along the world lines of matter in their positive direction would yield a coordinate system with the property that the $0^{\text {th }}$ coordinate always increases if one moves in a positive time-like direction, in contradiction to (6), which implies that all coordinates of the initial and the end point of a time-like line are equal in certain cases.

Property (5), for an open time coordinate, is an immediate consequence of the existence of closed time-like lines; for a closed time coordinate it follows from the fact that the subspaces $t=$ const. would contradict property (7) (as can easily be shown owing to the simple connectivity of $S$ ).

In order to prove property (8), let $U$ be an element of $\sum$; then $U$ intersects the subspace $S_{0}$ of $S$ defined by $x_{3}=0$ in a surface $V$ (for it has one point in common with each $x_{0}$-line situated on $S_{0}$ ). Now, according to what was proved, $V$ cannot be orthogonal on all $x_{0}$-lines

${ }^{10}$ This computation is rather cumbersome. It is simpler to derive both forms of the linear element independently from each other from the geometrical meaning of $S$ given below. The first form is obtained by taking for the $x_{1} x_{2}$-space of the coordinate system the point set corresponding to any two-parametric subgroup of the multiplicative group of the hyperbolic quaternions as defined in footnote 14 . in $S_{0}$. So let $l$ be an $x_{0}$-line in $S_{0}$ on which it is not orthogonal, and $P$ the point of intersection of $V$ and $l$. Then by rotating $S_{0}$ around $l$ (and every $S_{b}$ defined by $x_{0}=b$ by the same angle around the $x_{0}$-line obtained from $l$ by the translation $x_{3}{ }^{\prime}=x_{3}+b$ ), $U$ goes over into a three-space different from $U$, but passing through $P$, hence not contained in $\sum$, since the elements of $\sum$ were assumed to be mutually exclusive. Hence $\sum$ goes over into a system different from $\sum$.

\section{SOME ADDITIONAL THEOREMS AND CON- SIDERATIONS ABOUT THE SOLUTION}

I am mentioning without proof that, disregarding the connectivity in the large (which can be changed by identifying the points of certain point sets with each other), the solution given and Einstein's static universe are the only spatially homogeneous cosmological solutions with non-vanishing density of matter and equidistant world lines of matter. ${ }^{11}$

The space $S$ has a simple geometric meaning. It is the direct product of a straight line and the three-space $S_{0}$, defined by $x_{3}=0$; and $S_{0}$ is obtained from a space $R$ of constant positive curvature and signature +by stretching the metric ${ }^{12}$ in the ratio $\sqrt{2}: 1$ in the direction of a system of time-like Clifford parallels. ${ }^{13}$

This definition of $S_{0}$ also leads to an elegant representation of its group of transformations. To this end we map the points of $R$ on the hyperbolic quaternions $u_{0}+u_{1} j_{1}+u_{2} j_{2}+u_{3} j_{3}$ of positive absolute value ${ }^{14}$ by means of projective coordinates ${ }^{15} u_{0} u_{1} u_{2} u_{3}$ so chosen, that Klein's fundamental quadric takes on the form

11 There exist stationary homogeneous solutions in which the world lines of matter are not equidistant. They lead, however, into difficulties in consequence of the inner friction which would arise in the "gas" whose molecules are the galaxies, unless the irregular motion of the galaxies is zero and stays so.

${ }_{12} \mathrm{By}$ "stretching the metric in the ratio $\mu$ in the direction of the lines of a system $\pi$ "I mean that a new distance $P Q$ ' of neighboring points is introduced by the equation $\left(P Q^{\prime}\right)^{2}=P R^{2}+(\mu \cdot R Q)^{2}$, where $R$ is the foot of the perpendicular drawn from $P$ on the line of $\pi$ passing through $Q$; or, in other terms : $\left(d s^{\prime}\right)^{2}=d s^{2}+\left(\mu^{2}-1\right)\left(v_{i} d x_{i}\right)^{2}$, where $v$ is the field of the tangent vectors of unit length of the lines of $\pi$.

${ }^{13}$ That is, a system of pairwise equidistant straight lines which for each point of space contains exactly one line passing through it.

${ }_{14}$ Here the $u_{i}$ are real numbers and the units $j_{n}$ are defined by $j_{1}=i_{1}, j_{2}=i \cdot i_{2}, j_{3}=i \cdot i_{3}$, where the $i_{n}$ are the units of the ordinary quaternions and $i$ the imaginary unit, which is assumed to commute with all $i_{n}$. The term "hyperbolic quaternions" occurs in the literature in a different sense, but the number system just defined evidently is what should be so-called. For: $\operatorname{norm}(u)=u \cdot \bar{u}$ $=u_{0}^{2}+u_{1}^{2}-u_{2}^{2}-u_{3}^{2}$, and moreover, the multiplicative group of these quaternions, if quaternions differing by a real factor are identified, is isomorphic with the group of transformations of the Lobatchefskian plane into itself. That the metric of $R$ remains invariant under the transformations given in the text follows immediately from the equation $\operatorname{norm}(u v)=\operatorname{norm}(u) \cdot \operatorname{norm}(v)$.

${ }_{15}$ It is to be noted, however, that there exist different topological forms of spaces of constant positive curvature and signature -1 , and that that form which can be represented in projective coordinates in a one-to-one manner does not lead exactly to the space $S$ defined before, but rather to a space obtained from $S$ by identifying any two points which are situated on the same line of the system $\pi$ and whose distance on that line is equal to a certain constant. A corresponding difference subsists for the groups of transformation. 
$u_{0}{ }^{2}+u_{1}{ }^{2}-u_{2}{ }^{2}-u_{3}{ }^{2}$. Then, any motion $u \rightarrow u^{\prime}$ of $R$ into itself can be represented in the form $u^{\prime}=p \cdot u \cdot 9$, where $p$ and 9 are hyperbolic quaternions of positive norm. A system $\pi$ of Clifford parallels can be represented by $\sigma^{\alpha} \cdot u$, where $\sigma$ is a hyperbolic quaternion depending on $\pi$ alone, and the individual lines of $\pi$ are obtained by assigning a fixed value to $u$ and varying $\alpha$ from $-\infty$ to $+\infty$. It follows that those motions of $R$ into itself which leave $\pi$ (and the orientation of its lines) invariant are represented by $u^{\prime}=\sigma^{\beta} \cdot u \cdot 9$, where $\beta$ varies over all real numbers and $g$ over all hyperbolic quaternions of positive norm. These motions, however, evidently form the four-parametric continuous group of transformations which carry $S_{0}$ into itself. The lines of $\pi$, of course, are the world lines of matter.

Evidently, in whatever ratio $\mu$ the metric of $R$ is stretched in the direction of the lines of $\pi$, the resulting space $R^{\prime}$ has rotational symmetry. Therefore, the contracted Riemann tensor of $R^{\prime} \times l$ ( $l$ being a straight line), if the coordinate system in the point considered is orthonormal, and its first basis vector $e^{(0)}$ has the direction of the $\pi$-lines, its last one $e^{(3)}$ the direction of $l$, has the form $\left(\begin{array}{ccc}a & & \\ b & 0 \\ 0 & b & \\ & & 0\end{array}\right)$, where $a$ and $b$ are functions of $\mu$. Computation shows that $\mu=\sqrt{2}$ yields $b=0$, i.e., $R_{i k}=a \cdot e_{i}{ }^{(0)} e_{k}{ }^{(0)}$, which makes it possible to satisfy the field equations in the manner described above.

As the the physical meaning of the solution proposed in this paper, it is clear that it yields no red shift for distant objects. For, by using the transformation (I) defined in the proof of the properties (1) and (2), one proves immediately that light signals sent from one particle of matter (occurring in the solution) to another one arrive with the same time intervals in which they are sent. For the period of rotation one obtains $2 \cdot 10^{11}$ years, if for $\rho$ the value of $10^{-30} \mathrm{~g} / \mathrm{cm}^{3}$ is substituted. Assuming galactical systems were formed by condensation of matter originally distributed uniformly, and taking for the ratio of contraction 1:200 (which is suggested by the observed average ratio of 1:200 between diameter and distance of galaxies), one obtains (using the law of conservation of angular momentum) for the average period of rotation of galactic systems $5 \cdot 10^{6}$ years. This number is of the correct order of magnitude, but, in view of the fact that this would have to be approximately the period of rotation in the outer parts of the nebulae, the observed value is found to be considerably larger. ${ }^{16}$ Of course such comparison with observation has very little significance before an expansion has been combined with the rotation. Moreover, an explanation would have to be found for the apparent irregularity of the distribution of the axes of rotation of the galaxies. This, however, is perhaps not impossible, since there exist various circumstances which would tend to blur the original order, or make it appear blurred, especially if the axes of rotation of matter in different places (unlike in the solution described above) were not parallel with each other. The radius of the smallest time-like circles, in the solution given in this paper, is of the same order of magnitude as the world radius in Einstein's static universe.

${ }^{16}$ From the numerical data which E. Hubble, Astrophys. J. 79, 74 (1934) gives about two galaxies of medium size follow periods of rotation of $2 \cdot 10^{7}$ and $7 \cdot 10^{7}$ years at a distance of about half the radius from the center. The period of rotation of the Andromeda Nebula in the central region is estimated at $1,5 \cdot 10^{7}$ years. 